\title{
Detecting pharyngeal post-swallow residue by ultrasound examination: a case series
}

\author{
Yuka Miura ${ }^{1,2}$, Gojiro Nakagami ${ }^{1}$, Koichi Yabunaka ${ }^{1}$, Haruka Tohara ${ }^{3}$, Koji Hara ${ }^{3}$, \\ Hiroshi Noguchi ${ }^{4}$, Taketoshi Mori ${ }^{4}$, Hiromi Sanada ${ }^{1}$
}

${ }^{1}$ Department of Gerontological Nursing/Wound Care Management, The University of Tokyo, ${ }^{2}$ Japan Society for the Promotion of Science, ${ }^{3}$ Gerodontology and Oral Rehabilitation, Department of Gerontology and Gerodontology, Graduate School of Medical and Dental Sciences, Tokyo Medical and Dental University, ${ }^{4}$ Department of Life Support Technology (Molten), The University of Tokyo, Tokyo, Japan.

\begin{abstract}
Aims: Prevention of aspiration pneumonia is a great concern in the era of global aging. The assessment of pharyngeal post-swallow residue is important because remaining food and liquid in the pharyngeal area has a possibility of flowing into the lower airway which can cause aspiration pneumonia. Ultrasound examination has been recently used to assess swallowing disorders because of its noninvasiveness. The aim of this study was to investigate the performance for detecting pharyngeal post-swallow residue using an ultrasound examination by comparing with those using a fiberoptic endoscopic evaluation of a swallowing study as a reference. Material and methods: A linear array transducer attached to the thyroid cartilage to visualize the vocal folds in the sagittal plane. The pharyngeal post-swallow residue in the ultrasound movie was interpreted as a misty hyperechoic area above the vocal folds that remained after swallowing. Nineteen ultrasound images simultaneously obtained with fiberoptic endoscopic evaluation of swallowing study images from nine participants (eight men) with a median age of 70 years at a dysphagia outpatient clinic were analyzed by the montage of swallowing examinations. Results: Misty hyperechoic areas above the vocal folds that remained after swallowing were detected in 10 ultrasound images. Eight ultrasound images out of ten correctly detected pharyngeal post-swallow residue. The sensitivity for detecting pharyngeal post-swallow residue of 19 images from nine participants by ultrasound examination was $62 \%$, and the specificity was $67 \%$. Conclusions: The proposed detection method of pharyngeal post-swallow residue by ultrasound examination enabled the real-time assessment for swallowing of people with dysphagia. Ultrasound assessment-based daily swallowing care which detects pharyngeal residue will be useful for preventing aspiration pneumonia.
\end{abstract}

Keywords: aspiration pneumonia, deglutition disorders, residue, ultrasonography

\section{Introduction}

In this era of global population aging, pneumonia in adults is a greater concern than it has been in the past. In Japan, which has the fastest growing aged population, pneumonia is the third leading cause of death [1]. Most com-

Received 01.02.2015 Accepted 30.04.2016

Med Ultrason

2016, Vol. 18, No 3, 288-293

Corresponding author: Hiromi Sanada

Department of Gerontological Nursing/

Wound Care Management

Graduate School of Medicine,

The University of Tokyo,

7-3-1 Hongo, Bunkyo-ku,

Tokyo 113-0033, Japan

Phone/Fax: +81-3-5841-3442,

E-mail: hsanada-tky@umin.ac.jp munity-onset pneumonia in elderly people, which includes both community-acquired pneumonia and health care-associated pneumonia, is related to aspiration pneumonia [2]. The estimated annual number of cases of aspiration pneumonia in Japan was 630,000 in 2013 [2]. Strategies are required to prevent aspiration pneumonia in the aging society.

Swallowing disorders including aspiration and pharyngeal post-swallow residue are the main risk factors of aspiration pneumonia [3]. The pharyngeal post-swallow residue is the presence of food or liquid in the hypopharynx that is not entirely eliminated by swallowing [4]. The pharyngeal post-swallow residue is a predictor of aspiration that causes aspiration pneumonia because the residue suggests impairment of the driving forces of the oropharyngeal bolus and reduced swallowing efficiency [5]. The accumulated pharyngeal post-swallow residue 
sometimes flows into the airway without cough reflex in people with dysphagia. The presence of pharyngeal postswallow residue increases the risk of aspiration more than 2.75 times compared with the absence of residue [6]. Therefore, assessing pharyngeal post-swallow residue is important to provide appropriate swallowing care to prevent aspiration pneumonia.

Clinicians usually perform a videofluoroscopic swallowing study (VFSS) or fiberoptic endoscopic evaluation of swallowing (FEES) to assess pharyngeal post-swallow residue $[4,7]$. With VFSS, the pharyngeal residue is visualized as a shadow in the hypopharynx after the participant swallows a contrast agent such as barium sulfate. With FEES, food and liquid in the hypopharynx can be observed directly through an endoscope. The vallecular space, that is the space between the epiglottis and base of the tongue, is one of the hypopharyngeal areas where the pharyngeal post-swallow residue is often detected [8]. Residue in this area is difficult to detect just by visual examination; therefore, some imaging examinations such as VFSS and FEES are required. However, these invasive methods are not adequate as an assessment in daily mealtimes.

A few researchers have tried to detect pharyngeal post-swallow residue using acoustic measurements as less invasive way $[9,10]$. They tried to assess pharyngeal post-swallow residue that was detected through VFSS or FEES from the voice or breath sounds after swallowing. These studies reported that it was difficult to achieve both good sensitivity and specificity. It was difficult to classify normal and abnormal status in the hypopharynx based on acoustic measurements as many noises interfere with the evaluation. Therefore, these methods required acoustic analysis; thus, they were not real-time assessment methods of residue. Visualization of the internal hypopharynx will provide sufficient real-time information and will improve sensitivity and specificity for detecting pharyngeal post-swallow residue.

Ultrasound (US) examination appears to be an ideal imaging method for detecting pharyngeal post-swallow residue because it is noninvasive and can be performed at the bedside. In our previous study we showed that it was possible to distinguish aspirated boluses from surrounding tissues based on the differences in the characteristics of their movements in US movies [11,12]. Distinguishing pharyngeal post-swallow residue from the surrounding tissues will be more difficult than distinguishing aspirated boluses from the surrounding tissues because there are no differences between remaining food and liquid boluses and surrounding tissues with respect to the characteristics of their movement. However, the acoustic impedance of food and liquid will be different from that of surrounding tissues. Therefore, we hypothesized that pharyngeal post-swallow residue can be detected as the region with a different brightness from the surrounding tissues, especially the vocal folds and pharyngeal muscles. The aim of this study was to investigate the performance for detecting pharyngeal post-swallow residue using ultrasound examination by comparing with those using FEES as a reference.

\section{Material and methods}

\section{Study design}

All the data were obtained from a cross-sectional study that was conducted from August to November in 2012. This study used US images that were obtained in the previous study to develop a method to detect aspiration by US examination [12].

\section{Participants and setting}

The data were collected at a dysphagia outpatient clinic of a general hospital in Chiba Prefecture, Japan. Participants who underwent US examination simultaneously with FEES were included in this study. Participants with conditions that made it difficult to attach a transducer in the appropriate position were excluded from the study. Examinations of swallowing of high viscosity materials including liquid with thickening agent and paste food were excluded from the analysis. Participants with swallowing problems for high viscosity usually shows abnormal swallowing such as coughing, therefore; they do not require an imaging examination. The study protocol was approved by the Ethics Committee of the Graduate School of Medicine, The University of Tokyo [\#3260-(1)]. Written informed consent was obtained from all participants or their proxies.

\section{Ultrasound examination}

The US examinations were performed simultaneously with a FEES. The type of the test boluses, the number of the measurements and the order of the measurements were determined based on each participant's swallowing ability assessed before the examinations by the experienced dentists who performed FEES. The test bolus included foods and liquids with different viscosities. A portable ultrasound (M-Turbo; Sonosite, Bothwell, WA, USA) with a 5 to $15 \mathrm{MHz}$ (HFL50x) linear array transducer was used for the examination. US movies of 6-30 s of swallowing were obtained. The head and neck positions of the participants were not fixed so that they could swallow boluses in a way that was most comfortable for them. A US operator asked the subjects to speak before the examination in order to visualize the vibrations of the vocal folds in the US images. A transducer was attached on the thyroid cartilage to visualize the vocal folds in the sagittal plane in the 
cervical area (fig 1a). Subjects were instructed to keep their eyes front to reduce the artifacts from neck movement during the examination. A US operator controlled the pressure exerted by the transducer in order not to disturb the participants' swallowing during the examination. The depth of the visualization was kept at 40 $\mathrm{mm}$ from the skin surface to include the vocal folds as a landmark and the hypopharyngeal and tracheal area. The echo gain and dynamic range were adjusted to a proper level for each measurement to clearly visualize the target area. Other settings including focus, frequency, and zoom were predetermined and maintained at a constant value during the examination. The pharyngeal post-swallow residue was interpreted as a misty hyperechoic area above the vocal folds that remained after swallowing (fig 1b). A researcher who was blinded to the information of the participants and the examinations evaluated the presence or absence of pharyngeal postswallow residue from the US movies.

\section{Fiberoptic endoscopic evaluation of swallowing}

FEES was performed by experienced dentists, and the results were used as a reference in this study. A fiber-

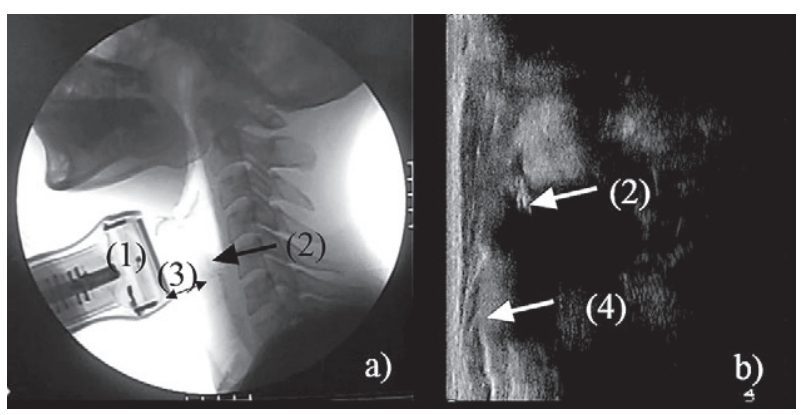

Fig 1. The scanning method of US examination for the detection of pharyngeal post-swallow residue: a) US transducer was attached in the sagittal plane above the thyroid cartilage; b) The vocal folds that were depicted as vibrated hyperechoic objects were used as a landmark during US examination for detecting pharyngeal post-swallow residue. (1) The US transducer, (2) vocal folds, (3) the trachea, (4) the tracheal wall. Upper side of the images represented the cranial side. The left side of the US images were skin surface, where, transducers were attached. US: ultrasound. optic fluorescence imaging system (FNL-10RBS; PENTAX Medical, Ontario, Canada) was used for the FEES. A small amount of $2 \%$ lidocaine was applied to the tip of the fiberscope to enhance exam tolerability and not to impair swallowing ability. A previous study reported that a small amount of lidocaine did not affect the swallowing ability [13]. A coloring agent was introduced to enhance the visualization of liquid in the FEES. An experienced dentist who was blinded to the results of the US examination evaluated the presence or absence of the pharyngeal post-swallow residue in the vallecular space on the FEES images. He was also blinded to the information of the participants and the examinations.

\section{Data analyses}

The FEES results were used as a reference for calculating the sensitivity and specificity of the US examination for detecting pharyngeal post-swallow residue. The evaluation was performed for each measurement, not for each participant as most of the participants underwent multiple measurements that included different types of test boluses. Fisher's exact test was used for the analysis. The $\mathrm{p}$ value was set at 0.05 for statistical significance. All analyses were conducted using STATA, version 14 (STATA Corp., College Station, TX, USA).

\section{Results}

Twenty-five US and 25 FEES images were simultaneously obtained. Owing to poor image quality, two FEES images were difficult to evaluate and thus, excluded from the analysis. Four examinations of high viscosity swallowing were excluded from the analysis. Finally, 19 images from nine participants (eight men) with a median age of 70 years were analyzed (Table I). The residue was found in eight participants and aspiration was found in four participants by FEES. The vibration of the vocal folds and swallowing reflex were detected in all images of US examination and FEES. Figure 2 shows a montage of a complete swallowing exam of US examination and FEES. Misty hyperechoic

Table I. Characteristics of the participants.

\begin{tabular}{lllll}
\hline Case & Age/ sex & Diseases & Aspiration detected by FEES & Residue detected by FEES \\
\hline 1 & $70 / \mathrm{M}$ & Stroke & - & + \\
2 & $60 / \mathrm{M}$ & Stroke & - & + \\
3 & $60 / \mathrm{M}$ & Stroke & + & + \\
4 & $80 / \mathrm{M}$ & Amyotrophic lateral sclerosis & + & + \\
5 & $60 / \mathrm{M}$ & Stroke & + & + \\
6 & $62 / \mathrm{F}$ & No diseases & - & + \\
7 & $75 / \mathrm{M}$ & Pneumonia & - & + \\
8 & $76 / \mathrm{M}$ & Parkinson's disease, stroke & + & + \\
9 & $70 / \mathrm{M}$ & Stroke & - & \\
\hline
\end{tabular}


areas above the vocal folds that remained after swallowing were detected in 10 US images. Eight US images out of ten were correctly detected pharyngeal post-swallow residue. The sensitivity for detecting pharyngeal postswallow residue of 19 images from nine participants by US examination was $62 \%$, and the specificity was $67 \%$ (Table II). Two typical cases for which US examination detected pharyngeal post-swallow residue are described in figure 3 and 4.
Table II. Results of the detection of pharyngeal post-swallow residue.

\begin{tabular}{llll}
\hline $\mathbf{N = 1 9}$ & & FEES & \\
& & Presence & Absence \\
\hline US & Detected & 8 & 2 \\
& Not detected & 5 & 4 \\
Sensitivity $=62 \%$, specificity $=67 \%$ & & \\
\hline
\end{tabular}

Nineteen images from nine participants were analyzed; FEES: fiberoptic endoscopic evaluation of swallowing; US: ultrasound

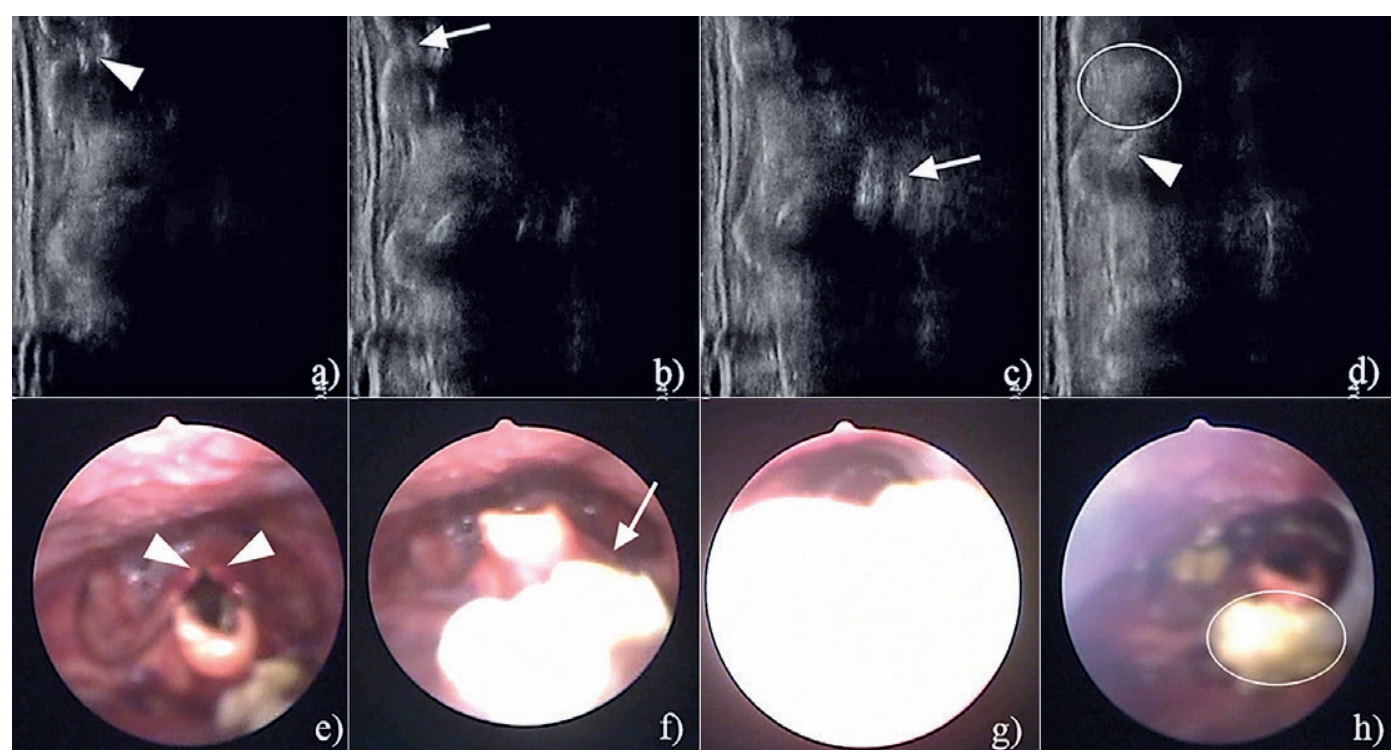

Fig 2. A montage of a US examination and FEES. The vibration of the vocal folds before swallowing examination was detected in the US image as hyperechoic spots (a). Swallowed boluses were observed as hyperechoic lines in the hypopharyngeal area (b). Swallowing reflex was detected as the flow of hyperechoic object in the esophageal area (c). After the swallowing reflex, remaining boluses in the hypopharyngeal area were observed as a hyperechoic area above the vocal folds (d). The vibration of the vocal folds and swallowed boluses were also detected in the FEES image (e,f). When the swallowing reflex occurred, the pharynx squeezed around the endoscope's camera and caused a white colored reflection on the FEES image (g). The FEES image showed a residue of boluses in the vallecular space after swallowing (h). Arrow heads represent vocal folds. Arrows represent food boluses. Circles represent pharyngeal post-swallow residue of food.

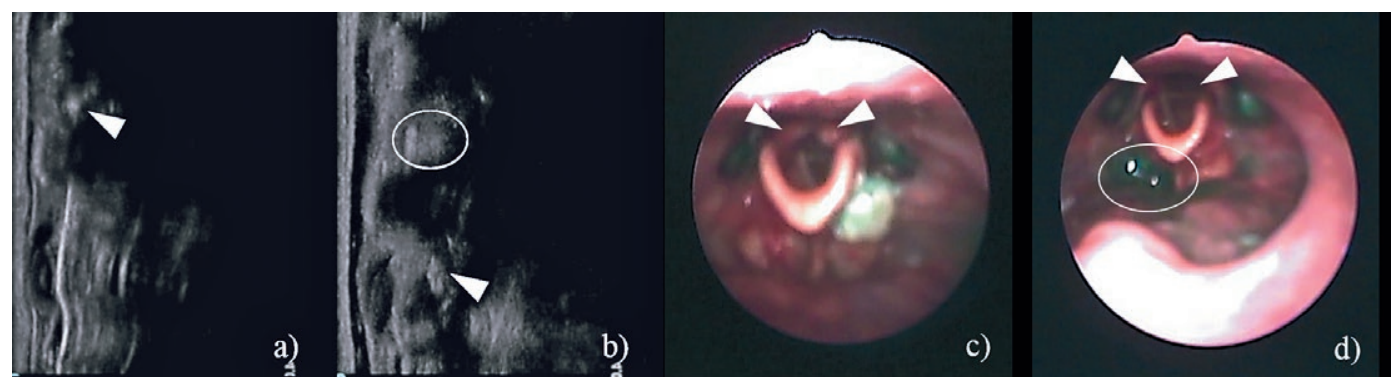

Fig 3. Only residue detected. A 75-year-old man with a history of aspiration pneumonia underwent FEES. His usual meals consisted of soft food, and he stated that he did not have a cough during mealtime. The participant was asked to swallow a colored liquid with low viscosity with his head oriented up in a $90^{\circ}$ angle. US images depicted the vocal folds as a hyperechoic spot before swallowing (a). After the swallowing reflex occurred, a misty hyperechoic area was observed above the vocal folds (b). Before the swallowing reflex, FEES images showed no residue of green liquid with a coloring agent in the vallecular space (c). FEES images showed a residue of colored liquid in the vallecular space after swallowing (d). Aspiration was not detected in the FEES images. Arrow heads represent vocal folds. Circles represent pharyngeal post-swallow residue of liquid. 


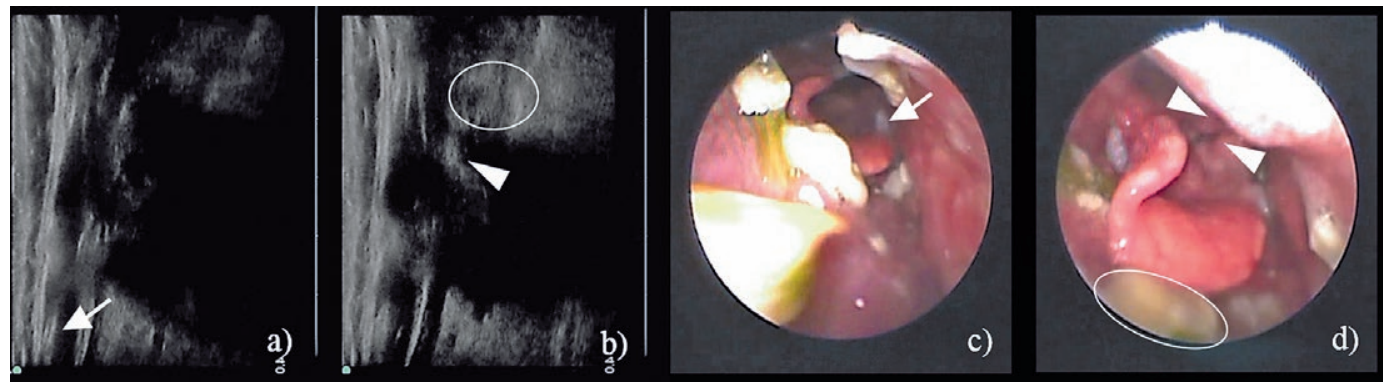

Fig 4. Both aspiration and residue detected. A 76-year-old man with a history of Parkinson's disease and stroke underwent FEES to evaluate changes in his swallowing ability. He complained of increased sputum after he had a fever 2 weeks earlier. He was eating a low-viscosity diet. He used a thickening agent to prevent aspiration when he was drinking liquid. The participant was asked to swallow rice and vegetables with low viscosity with his head oriented up in a $90^{\circ}$ angle. Aspirated rice grains were observed as hyperechoic narrow objects along the tracheal wall moving into the trachea in the US examination (a). The US images also showed the vocal folds with a hyperechoic misty area after he swallowed rice porridge and minced vegetables (b). The FEES images showed aspiration of rice during the swallowing (c) and the remaining boluses of rice and vegetables in the vallecular space after swallowing (d). Arrows represent aspirated boluses. Arrow heads represent vocal folds. Circles represent pharyngeal post-swallow residue.

\section{Discussions}

To the best of our knowledge, this is the first study showing that pharyngeal post-swallow residue can be detected by US examination. In addition, the performance of the US examination in detecting pharyngeal post-swallow residue was evaluated in comparison with FEES.

There are some previous studies that clearly visualized the oropharyngeal anatomy including vocal folds and boluses in the oropharyngeal area by US examination [14-17]. The finding in this study that the pharyngeal residue was detected as a misty hyperechoic area above the vocal folds in US images that could be distinguished from the soft tissues around the vocal folds was consistent with these previous studies. Pharyngeal residue seems to be more difficult to detect by US examination than aspiration in the tracheal area because residue does not show any characteristic movement during swallowing. We focused on the vocal folds, which are close to the vallecular space to detect pharyngeal post-swallow residue. The vallecular space is one of the most common hypopharyngeal areas where boluses remain after swallowing. Thus, we succeeded in detecting pharyngeal post-swallow residue as a misty hyperechoic area above the vocal folds in US images.

The advantage of the detection of pharyngeal postswallow residue using US examination is that the realtime assessment of various types of foods and liquids is possible with acceptable sensitivity and specificity based on the imaging method. The sensitivity and specificity of US for detecting residue were not always higher than the results of previous methods $[9,10]$ which aimed to detect dysphagia including pharyngeal post-swallow residue by voice or breath sounds after the swallowing ( $62 \%$ vs $8 \%-92 \%, 67 \%$ vs $59 \%-86 \%$ ). Because previous methods only detected residue from liquids, the sensitivity and specificity of residue from various types of foods were not verified. US examination indicated the possibility of usefulness for applying it to mealtimes for the detection of pharyngeal post-swallow residue.

In our previous study, which aimed at detecting aspiration, we also found a higher disagreement between US examination and FEES for low-viscosity boluses [12]. The size of the boluses may affect the visualization of pharyngeal post-swallow residue. The amount of residue of low-viscosity boluses will be small because they spread fast, and thus, it is difficult to visualize as well as aspirated boluses by US examination. The sensitivity and specificity of an US examination for detecting pharyngeal post-swallow residue were lower than those for detecting aspirated boluses. Because the information of movement was not available for detecting residue, the evaluation of residue would be more difficult than that of aspiration, which showed characteristic movement of aspirated boluses for evaluation.

The main limit of the study is that we did not included subjects with anatomical different features in the oropharyngeal area, such as patients with a tracheostomy and laryngectomy. These subjects would show different oropharyngeal US images from this study, therefore, a further study is required to investigate the application of the US examination with a larger sample size, the small number of the studied subjects being another limitation of the study.

Many healthcare providers have tried to reduce residue in clinical settings. The viscosity of the food and liquid is known to influence the amount of oropharyn- 
geal residue [18]. Therefore, healthcare providers often modify the viscosity of the food and liquid to reduce pharyngeal post-swallow residue when the residue was detected. Moreover, some types of swallowing exercises are reported to be effective for reducing the amount of pharyngeal post-swallow residue $[19,20]$. The type of the swallowing exercise for reducing the amount of pharyngeal post-swallow residue is different from that of the exercise for reducing the amount of aspiration. The exercises for reducing the amount of pharyngeal postswallow residue focus on the movement of the pharyngeal constrictor $[19,20]$. On the other hand, the reduction of the amount of aspiration focuses on the glottal closure [21]. The important point is that these strategies to reduce pharyngeal post-swallow residue are different from those to reduce aspiration. This proposed US method will contribute to the prevention of aspiration pneumonia because there has been no imaging method to detect pharyngeal post-swallow residue during daily mealtime.

In conclusion, this study was the first attempt to detect pharyngeal post-swallow residue by US examination. In US images, the pharyngeal post-swallow residue is defined as a misty hyperechoic object above the vocal folds after swallowing. Since both pharyngeal postswallow residue and aspiration can be risk factors of aspiration pneumonia, the detection method based on US examination will be able to propose a comprehensive US assessment-based swallowing care recommendation to prevent aspiration pneumonia. Further study with subjects with variety of diseases is still required to generalize the method of US examination to a wider population.

Acknowledgment: This study was funded by a Grant-in-aid for Challenging Exploratory Research from the Japan Society for the Promotion of Science to Hiromi Sanada (Grant No. 23659999) and by a Grant-in-aid for the Japan Society for the Promotion of Science to Yuka Miura (Grant No. 25-2360).

\section{Conflict of interest: none}

\section{References}

1. Morimoto K, Suzuki M, Ishifuji T, et al. The burden and etiology of community-onset pneumonia in the aging Japanese population: A multicenter prospective study. PLoS One 2015; 10: e0122247.

2. Ministry of Health Labour and Welfare. Vital statistics. [http://www.mhlw.go.jp/toukei/saikin/hw/jinkou/geppo/ nengai14/index.html], Accessed 3 Feb 2016.

3. Marik PE, Kaplan D. Aspiration pneumonia and dysphagia in the elderly. Chest 2003; 124: 328-336.
4. Murray J, Langmore S, Ginsberg S, Dostie A. The significance of accumulated oropharyngeal secretions and swallowing frequency in predicting aspiration. Dysphagia 1996; 11: 99-103.

5. Kelly AM, Macfarlane K, Ghufoor K, Drinnan MJ, LewGor S. Pharyngeal residue across the lifespan: A first look at what's normal. Clin Otolaryngol 2008; 33: 348-351.

6. Han TR, Paik NJ, Park JW. Quantifying swallowing function after stroke: A functional dysphagia scale based on videofluoroscopic studies. Arch Phys Med Rehabil 2001; 82: 677-682.

7. Leder SB, Murray JT. Fiberoptic endoscopic evaluation of swallowing. Phys Med Rehabil Clin N Am 2008; 19: 787-801.

8. Steele CM, Bailey GL, Chau T, et al. The relationship between hyoid and laryngeal displacement and swallowing impairment. Clin Otolaryngol 2011; 36: 30-36.

9. Waito A, Bailey GL, Molfenter SM, Zoratto DC, Steele CM. Voice-quality abnormalities as a sign of dysphagia: Validation against acoustic and videofluoroscopic data. Dysphagia 2011; 26: 125-134.

10. Yamashita M, Yokoyama K, Takei Y, et al. Acoustic characteristics of voluntary expiratory sounds after swallow for detecting dysphagia. J Oral Rehabil 2014; 41: 667-674.

11. Miura Y, Nakagami G, Tohara H, et al. B-mode video ultrasonography for detecting aspiration: Two cas studies. J Soc Nurs Pract 2014; 27: 42-49.

12. Miura Y, Nakagami G, Yabunaka K, et al. Method for detection of aspiration based on b-mode video ultrasonography. Radiol Phys Technol 2014; 7: 290-295.

13. O'Dea MB, Langmore SE, Krisciunas GP, et al. Effect of Lidocaine on Swallowing During FEES in Patients With Dysphagia. Ann Otol Rhinol Laryngol 2015; 124: 537-544.

14. Hu Q, Zhu SY, Luo F, Gao Y, Yang XY. High-frequency sonographic measurements of true and false vocal cords. J Ultrasound Med 2010; 29:1023-1030.

15. Gervasio A, D’Orta G, Mujahed I, Biasio A. Sonographic anatomy of the neck: The suprahyoid region. J Ultrasound 2011; 14: 130-135.

16. Casas MJ, Seo AH, Kenny DJ. Sonographic examination of the oral phase of swallowing: Bolus image enhancement. J Clin Ultrasound 2002; 30: 83-87.

17. Geddes DT, Chadwick LM, Kent JC, Garbin CP, Hartmann PE. Ultrasound imaging of infant swallowing during breast-feeding. Dysphagia 2010; 25: 183-191.

18. Steele CM, Alsanei WA, Ayanikalath S, et al. The influence of food texture and liquid consistency modification on swallowing physiology and function: A systematic review. Dysphagia 2015; 30: 2-26.

19. Wada S, Tohara H, Iida T, Inoue M, Sato M, Ueda K. Jawopening exercise for insufficient opening of upper esophageal sphincter. Arch Phys Med Rehabil 2012; 93: 1995-1999.

20. Don Kim K, Lee HJ, Lee MH, Ryu HJ. Effects of neck exercises on swallowing function of patients with stroke. J Phys Ther Sci 2015; 27: 1005-1008.

21. Rajaei A, Barzegar Bafrooei E, Mojiri F, Nilforoush MH. The occurrence of laryngeal penetration and aspiration in patients with glottal closure insufficiency. ISRN Otolaryngol 2014; 2014: 587945. 